\title{
Neutron stars: history of the magnetic field decay
}

\author{
Andrei P. Igoshev and Alexander F. Kholtygin \\ Institute of Astronomy, Saint Petersburg State University \\ email: ignotur@gmail.com
}

\begin{abstract}
Using the data of the ATNF pulsar catalog we study the relation connected the real age $t$ of young neutron stars (NS) and their spin-down age $\tau$. We suppose that this relation is independent from both initial period of the NS and its initial surface magnetic field, and that the laws of the surface magnetic field decay are similar for all NSs in the Milky Way. We further assume that the birth-rate of pulsars was constant during at least last 200 million years. With these assumptions we were able to restore the history of the magnetic field decay for the galactic NSs. We reconstruct the universal function $f(t)=B(t) / B_{0}$, where $B_{0}$ is the initial magnetic field and $B(t)$ is the magnetic field of NS at the age $t$. The function $f(t)$ can be fitted by a power law with power index $\alpha=-1.17$.
\end{abstract}

Keywords. methods: data analysis, methods: statistical, stars: neutron, magnetic fields, pulsars: general

\section{Introduction}

Magnetic fields determine observational manifestations of neutron stars. Soon after the discovery of the first radio pulsars, the decay of their magnetic field was supposed by Ostriker \& Gunn (1969). Therefore comprehension of magnetic fields behavior aids not only in more accurately finding real pulsar ages, but also helps us understand the physical processes inside isolated neutron stars.

First of all we carefully consider behavior of spin-down age of radio pulsar:

$$
\tau=\frac{P}{\dot{P}} \text {. }
$$

Here $P$ is period of pulsar and $\dot{P}$ is its derivative. It appears that $\tau$ is highly sensitive to changes in magnetic fields of NS. We find that it is possible to introduce an additional real pulsar age estimation based on the counts of pulsars with fixed spin-down age $\tau$. If we suppose that the law governing magnetic-field decay is identical for all pulsars, and can be present by the formula $B(t)=B_{0} f(t)$, then it is possible to restore the dimensionless factor $f(t)$ irrespectively of initial pulsar period distribution and initial distribution of their magnetic fields. This factor can be expressed as

$$
f(t)=\exp \left(\int_{0}^{t} \frac{d t^{\prime}}{2 \tau\left(t^{\prime}\right)}\right) / \sqrt{\tau(t)}
$$

The algorithm and detailed description of our method of restoring the function $f(t)$ is described in Igoshev (2012). Here we discuss the effects of the observational selection.

\section{Selection effects}

Results of restoring the function $f(t)$ by our method might be distorted by the significant observational selection effects. In fact, we observe only a small part of all galactic 
pulsars. And we can not be sure exactly that this small part correctly reproduces characteristics of the whole galactic ensemble of the NSs. Indeed, selection effects are able to hide some group of pulsars from our attention. In this case the selection effects can change an average and a variance of the parameters of the whole ensemble of the NS distribution.

On the other hand it is difficult to estimate this selection effects theoretically because of our poor understanding of the radiation mechanism for pulsars. Therefore, we will carefully analyze the observational data. We select the isolated radio-pulsars excluding the millisecond pulsars from the ATNF pulsar catalog by Manchester et al. (2005) $\dagger$. We divide them into five groups, where the spin-down age $\tau$ is expressed in years: $\tau \in$ $\left[700-1.2 \cdot 10^{5}\right], \tau \in\left[1.2 \cdot 10^{5}-4.5 \cdot 10^{5}\right], \tau \in\left[4.5 \cdot 10^{5}-9.6 \cdot 10^{5}\right], \tau \in\left[9.6 \cdot 10^{5}-1.6 \cdot 10^{6}\right], \tau \in$ $\left[4 \cdot 10^{6}-6 \cdot 10^{6}\right]$ and plot their cumulative distribution over their distance from the Sun in Fig. 1. It is clear that for pulsars of middle ages $\tau \in\left[1000,1.6 \cdot 10^{6}\right]$ the radial distributions are similar. The Kolmogorov-Smirnov test confirms, with a probability of more than $99 \%$, that these four samples can be described by a single distribution function. On the other hand the radial distribution of the group of older pulsars with $\tau \in\left[4 \cdot 10^{6}, 6 \cdot 10^{6}\right]$ years is different. Therefore, our conclusions below are correct only for pulsars from the four first age intervals.

Let us consider a cylinder in the Galaxy, centered on the Sun, with a radius $10 \mathrm{kpc}$ and with its axis normal to the Galactic plane. Our selection is similar to those by Lyne et al. (1998), but with a larger cylinder radius. As we study the relative quantities, the total number of objects which fall in the cylinder is not important.

We can show that the observational selection which does not conceal pulsars with certain ages is harmless for our analysis. Let us divide our cylinder with $10 \mathrm{kpc}$ radius into two parts: the inner cylinder A with radius $1.5 \mathrm{kpc}$ and remained part B of the large cylinder. The objects in cylinder A are belong to the local pulsar population. Designate the number of pulsars with spin-down ages in the interval $[\tau, \tau+\Delta \tau]$ inside the cylinder $\mathrm{A}$ as $n_{\alpha}$ and as $n_{\beta}$ for those which are in part $\mathrm{B}$ of the large cylinder. For second interval of the spin-down ages $\left[\tau^{\prime}, \tau^{\prime}+\Delta \tau\right]$ we designate the corresponding values as $n_{\gamma}$ in $\mathrm{A}$ and $n_{\delta}$. As it known from paper by Lyne et al. (1998) the observational selection effects are weak for object in the cylinder A. Therefore, we can estimate the ratio $n_{\alpha} / n_{\gamma}$ with high level of confidence. As it was discussed above the radial distribution function for pulsars of different spin-down ages are similar. It means that

$$
\frac{n_{\beta}}{n_{\alpha}}=\frac{n_{\delta}}{n_{\gamma}}
$$

Gathering the pulsars from parts A and B of the large cylinder together we can write the next relation:

$$
\frac{n_{\alpha}+n_{\beta}}{n_{\gamma}+n_{\delta}}=\frac{n_{\alpha}\left(1+n_{\delta} / n_{\gamma}\right)}{n_{\gamma}\left(1+n_{\delta} / n_{\gamma}\right)}=\frac{n_{\alpha}}{n_{\gamma}}
$$

We find that the count of pulsars in the large cylinder does not change the result. In the other hand, the larger number of pulsars located in the large cylinder allows us to make more precise conclusions.

\section{Results}

A new statistical method which allows us to restore history of magnetic fields decay for middle age neutron stars was developed by Igoshev (2012). This method was applied to

$\dagger$ http://www.atnf.csiro.au/research/pulsar/psrcat/ 


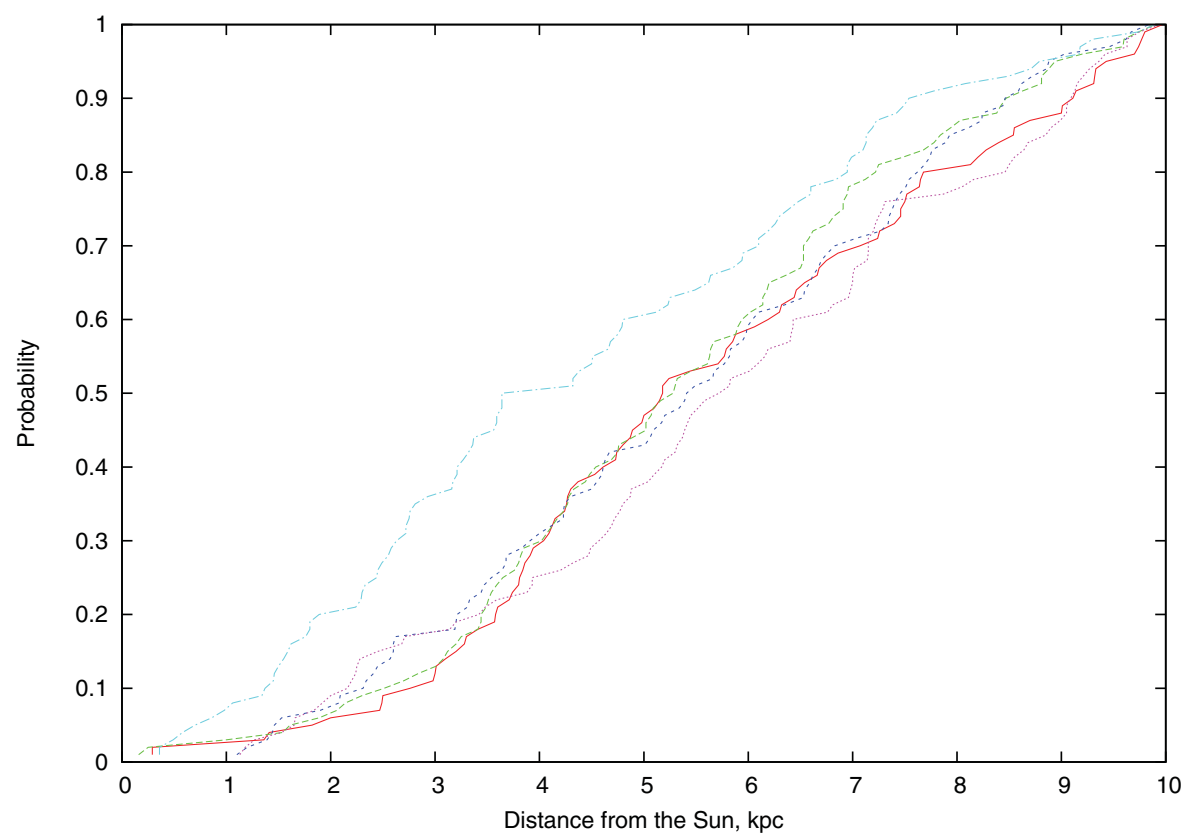

Figure 1. Cumulative distribution of the pulsars over distances from the Sun. The electronic version of article contains color figures. Here red solid line corresponds to ages $\tau \in\left[700,1.2 \cdot 10^{5}\right]$ years, green dashed line - to $\tau \in\left[1.2 \cdot 10^{5}, 4.5 \cdot 10^{5}\right]$ years, blue short-dashed line - to $\tau \in\left[4.5 \cdot 10^{5}, 9.6 \cdot 10^{5}\right]$ years, violet dotted line - to $\tau \in\left[9.6 \cdot 10^{5}, 1.6 \cdot 10^{6}\right]$ years and sky blue dashed and dotted line - to $\tau \in\left[4 \cdot 10^{6}, 6 \cdot 10^{6}\right]$ years. Each interval of ages includes about of 100 pulsars.

a real sample of radio pulsars from the ATNF catalog. We find that the surface magnetic fields of middle age radio pulsars decay following a modified power law:

$$
f(t)=\left(\left(a \frac{t}{t_{0}}\right)^{\gamma}+c\right)^{-1}
$$

with parameters $\gamma=1.17, a=0.034, c=0.84$ and $t_{0}=10^{4}$ years. It was found that observational selection is negligible when we take into consideration only part of all galactic volume. This effect is connected with similarity of radial distribution for radio pulsars of different ages in range $\left[700-1.6 \cdot 10^{6}\right]$ years.

We also estimate the pulsar birthrate supposing that pulsars with the spin-down age over $4 \cdot 10^{4}$ years have not experienced significant magnetic-field decay. This produces a birth rate of about 2.9 pulsars per century.

\section{References}

Manchester, R. N., Hobbs, G. B., Teoh, A., \& Hobbs, M. 2005 AJ, 129, 1993

Lyne, A. G., Manchester, R. N., Lorimer, D. R., Bailes, M., D’Amico, N., Tauris, T. M., Johnston, S., Bell, J. F., \& Nicastro, L. 1998 MNRAS, 259, 743

Igoshev, A. P. 2012 ArXiv:1204.3445

Ostriker, J. P. \& Gunn, G. E. 1969 ApJ, 157, 1395 